\title{
The impact of radical pelvic radiotherapy on nutrient intake
}

\author{
H. E. Armitage ${ }^{1}$, C. McGough ${ }^{2}$, L. Wedlake ${ }^{2}$, K. Whelan ${ }^{1}$ and H. J. N. Andreyev ${ }^{2}$ \\ ${ }^{1}$ Nutritional Sciences Division, King's College London, Stamford Street, London SE1 9NH and ${ }^{2}$ Department of Medicine, \\ Royal Marsden Hospital, Fulham Road, London SW3 6JJ
}

Malignancy in the gynaecological, urological or lower gastrointestinal tract is responsible for $27 \%$ of all newly diagnosed cancer cases in the UK. Pelvic radiotherapy (PRT) is a cornerstone of current treatment, but in more than $90 \%$ of patients induces acute bowel symptoms, such as diarrhoea, faecal incontinence, abdominal discomfort and bloating due to underlying changes in intestinal physiology. This prospective, observational study aimed to characterise quantitative changes in nutrient intake and nutritional status at baseline and following the conclusion of 4-5 weeks of adjuvant or radical PRT in outpatients with pelvic malignancies.

At baseline (week 0) and at 4-5 weeks patients completed a non-weighed, 3-d food diary. Gastrointestinal symptoms were assessed using validated scoring tools and anthropometry was measured. Food diaries were analysed using Dietplan 6 software (see Table).

In total, twenty-three males and twenty-one females (mean age 61.3 (range 42-81) years) completed food diaries at both time points. Although at no nutritional risk at baseline (based on BMI), twenty-six patients (59.1\%) lost weight during treatment, with a mean weight loss of 0.61 (SD 1.83) $\mathrm{kg}(P=0.03)$.

\begin{tabular}{|c|c|c|c|c|c|}
\hline \multirow[b]{2}{*}{ Nutrient intake } & \multicolumn{2}{|c|}{ Baseline $(n$ 44) } & \multicolumn{2}{|c|}{ After pelvic radiotherapy $(n 44)$} & \multirow[b]{2}{*}{$P$ value* } \\
\hline & Mean & SD & Mean & SD & \\
\hline Energy $(\mathrm{kJ} / \mathrm{d})$ & 8422 & 2460 & 7699 & 2293 & 0.02 \\
\hline Carbohydrate $(\mathrm{g} / \mathrm{d})$ & 240.2 & 78.7 & 215.9 & 71.4 & 0.03 \\
\hline Protein $(\mathrm{g} / \mathrm{d})$ & 81.9 & 25.3 & 74.5 & 23.9 & 0.07 \\
\hline Fat $(\mathrm{g} / \mathrm{d})$ & 77.2 & 27.8 & 71.1 & 24.1 & 0.10 \\
\hline $\operatorname{NSP}(g / d)$ & 14.2 & 5.1 & 12.7 & 4.8 & 0.05 \\
\hline
\end{tabular}

* Means were compared using a paired $t$ test.

There was a significant reduction in mean daily energy intake between the two time-points but no significant change in protein, carbohydrate, fat or NSP fractions indicating a generalised reduction in nutrient intake. These findings are in agreement with those of Guren et al. ${ }^{(1)}$ who completed a comparable study in thirty-one rectal cancer patients.

The results suggest that negative energy balance in some patients arises because of a generalised reduction in dietary intake secondary to radiation-induced bowel-related side effects during pelvic radiotherapy. Future research should assess whether acute changes in dietary intake have longer-term consequences.

1. Guren MG, Tobiassen LB, Trygg KU, Drevon CA \& Dueland S (2006) Eur J Clinl Nutr 60, 113-119. 